\title{
VERDAD Y JUSTIFICACIÓN
}

\author{
ANa Rosa Pérez Ransanz \\ INSTITUTO de Investigaciones Filosóficas
}

UNAM

it is not easy to steer our thinking between the Scylla of a teleological metaphysics and the Charybdis of relativism.

W. Stegmüller

En su ensayo sobre William James, Ruth Anna y Hilary Putnam afirman lo siguiente: "Muy a menudo, el problema en filosofía es que un filósofo que sabe qué es lo que quiere negar siente que no puede hacer simplemente eso, sino que debe ofrecer una tesis 'positiva', y la tesis positiva es frecuentemente un desastre" (1990, p. 223). Teniendo presente esta sabia observación, sobre todo cuando se trata de un tema como la verdad, procuraré que la siguiente discusión de las ideas de Putnam tenga un carácter básicamente crítico y no propositivo.

Se podría decir que uno de los aspectos que más claramente distinguen una perspectiva externalista de una perspectiva internalista es la forma en que se asume la relación entre verdad y justificación. El filósofo de temperamento externalista parte de la intuición de que aquello que hace verdadera o falsa a una creencia debe ser algo distinto de las creencias mismas. El valor de verdad de las creencias, o de las proposiciones, depende de algo que es distinto de las razones que tenemos para creer. Podríamos tener las mejores razones, la mejor justificación a favor de una creencia y, sin embargo, la creencia podría ser falsa, pues la verdad depende de un mundo que está "ahí afuera", o como dice Putnam, de un "mundo ya hecho". De esta manera, el filósofo externalista separa nítidamente la noción de verdad de la noción de justificación, apoyándose en el supuesto de un mundo que existe y tiene una naturaleza determinada, independientemente del conocimiento que los sujetos tengan, o puedan tener, de él.

En la perspectiva internalista, en cambio, se parte de la intuición de que todos nuestros conceptos - incluyendo el de verdad - y todas nuestras creencias dependen fuertemente de las capacidades y recursos con los que contamos en tanto seres racionales y sensibles. De aquí que aquello que consideramos co- 
mo el mundo esté, al menos en parte, constituido por el conocimiento que tengamos de él (o según las distintas versiones: por nuestra capacidad de referirnos a él, por el poder sintetizador de la mente, por nuestra imposición de esquemas conceptuales, teorías, lenguajes, etc.). $Y$ de aquí que la verdad deba analizarse en términos de las razones que tenemos, o podemos tener, para creer. De esta manera, el filósofo internalista tiende a relacionar estrechamente verdad y justificación, tan estrechamente que en algunos casos, como es el de Putnam, concibe la verdad como un cierto tipo de justificación.

Este rápido boceto de las intuiciones básicas que distinguen los temperamentos filosóficos de los que habla Putnam, permite entrever por qué la perspectiva internalista es un suelo propicio para cultivar toda la variedad y grados de relativismos, lo cual explica la constante preocupación de Putnam por deslindar su internalismo de los relativismos fáciles y simplones del "todo se vale". Sin embargo, si bien queda claro por qué la concepción de Putnam evita el Escila del absolutismo metafísico - al rechazar como incoherente la idea del mundo como una totalidad de objetos independientes de la mente, que es descriptible por una teoría completa y verdadera-, por otra parte, me parece que la manera en que Putnam pretende salvar la noción de verdad, frente al Caribdis del relativismo, le genera un conflicto con su tesis de la relatividad conceptual -la cual implica un cierto tipo de pluralismo-, y además lo lleva a postular una noción que no corresponde a lo que entendemos por verdad. Veamos por qué.

Para Putnam "la verdad es una idealización de la aceptabilidad racional" (1981, p. 55), en el siguiente sentido. Lo que hace que un enunciado, o una teoría, sea racionalmente aceptable es, en gran parte, su coherencia y su ajuste: coherencia de nuestras creencias entre sí y con nuestras experiencias, "tal como esas experiencias son ellas mismas representadas en nuestro sistema de creencias" ( $c f r .1981$, p. 50 y pp. 54-55). La coherencia, entonces, está en la base de las condiciones de justificación por las cuales es aceptable una creencia. Pero "la verdad no puede ser simplemente aceptabilidad racional, por una razón fundamental: se supone que la verdad es una propiedad del enunciado [statement $]^{1}$ que no se puede perder, mientras que la justificación sí se puede perder" (1981, p. 55). "Un enunciado puede ser racionalmente aceptable en un tiempo, y sin embargo no ser verdadero" (1981, p. X).

Ahora bien, ¿cómo puede un internalista distinguir la verdad de la justificación, si la verdad está amarrada a las razones para creer? La manera que encuentra Putnam para mantener una liga fuerte entre verdad y justificación,

1 Traduzco 'statement' como 'enunciado', pues a pesar de la referencia que en castellano puede tener este término a entidades lingüísticas específicas, en general remite a algo intermedio entre las proposiciones - que como entidades abstractas pueden ser filosóficamente problemáticas- y las oraciones de un lenguaje específico. El enunciado expresaría el complejo de significados en el que pueden coincidir distintas oraciones específicas de lenguajes alternativos. 
sin tener que asimilar la primera a la segunda, es justamente idealizando la justificación. Notemos que el principal objetivo de esta estrategia es salvar la vieja intuición realista de que la verdad es una propiedad de los enunciados, que no pueden perder.

Pero, ¿cómo entender esta idealización de la justificación? En Reason, Truth and History (cfr. pp. 55-56) Putnam hace una analogía con las idealizaciones que se hacen en la ciencia, y dice que las "condiciones epistémicamente ideales" son como los "planos sin fricción", esto es, son algo que de hecho nunca podemos alcanzar. Sin embargo, el mismo Putnam menciona de pasada un rasgo de las condiciones ideales que, en mi opinión, debilita mucho la analogía y la vuelve más bien fuente de confusión: las condiciones epistémicas ideales no sólo son inalcanzables, sino que ni siquiera podemos estar seguros de qué tanto nos hemos acercado a ellas. Esto marca una diferencia importante con las idealizaciones de la ciencia, pues éstas tienen una utilidad efectiva justamente porque se puede determinar el grado de aproximación a ellas. Pero si esto no sucede con las condiciones epistémicas ideales, ¿qué otra utilidad pueden tener en el esclarecimiento de la noción de verdad?

Hasta donde sé, aparte de esta analogía no muy afortunada, y algunas declaraciones en el sentido de que él no pretende dar una definición de verdad, o que no cree "que podamos ni siquiera bosquejar una teoría de la justificación de hecho, y menos aún una teoría de la justificación idealizada", Putnam prácticamente no había dicho nada de las condiciones ideales que fuera más allá de lo que encierra la siguiente cita: "las dos ideas claves de la teoría de la verdad como idealización son 1 ) que la verdad es independiente de la justificación aquí y ahora, pero no independiente de toda justificación. Afirmar que un enunciado es verdadero es afirmar que podría ser justificado. 2) Es de esperar que la verdad sea estable o 'convergente'; si tanto un enunciado como su negación pudieran 'justificarse', aun cuando las condiciones fueran tan ideales como se pudiera esperar, no tiene ningún sentido pensar que el enunciado tiene un valor de verdad" (1981, p. 56).

Estando así las cosas, no deja de sorprender - por lo menos a mí y a algunos otros que habíamos tratado de entender esta cuestión- la forma tan llana y simple en que Putnam se refiere a las condiciones ideales, en el prefacio a Realism with a Human Face. En respuesta a la cantidad de malentendidos generados, Putnam ofrece la siguiente descripción: "Por una situación epistémica ideal quiero decir algo como esto: si yo digo 'hay una silla en mi estudio', una situación epistémica ideal sería estar en mi estudio con las luces encendidas o con luz de día entrando por la ventana, con ningún problema con mi vista, con una mente no confusa, sin haber tomado drogas o haber sido sometido a hipnosis, etcétera, y mirar y ver si ahí hay una silla" (1990, p. VIII). Con este ejemplo, Putnam quiere describir una situación epistémica suficientemente buena, con lo cual se aclara que el supuesto de las condiciones ideales 
simplemente pretende destacar el hecho de que hay mejores y peores situaciones epistémicas con respecto a los distintos enunciados particulares; no se trata de aludir, como se pensó, a una situación utópica de "ciencia acabada", donde se pudiera determinar el valor de verdad de cualquier enunciado. Afirmar que un enunciado es verdadero, dice Putnam, "es afirmar que podría ser justificado si las condiciones epistémicas fueran lo suficientemente buenas" (1990, p. VII).

Por cierto, me parece que los trabajos de Putnam de 1987 a la fecha reflejan un cambio de énfasis, aunque no de dirección, en su tratamiento de la verdad. Las intuiciones básicas del realismo interno siguen siendo las mismas, sólo que ahora parecen encontrar otra forma de expresión. En lugar de hablar de la justificación en términos de coherencia, o de la verdad como una coherencia ideal, o de las condiciones ideales como condiciones de racionalidad, Putnam carga ahora el acento en las condiciones de prueba o de verificación (tanto actuales como posibles), entendidas a la manera pragmatista, y destaca el papel de los intereses y los valores en cada contexto. Me pregunto si se trata del desplazamiento de un enfoque coherentista por uno pragmatista, o si Putnam piensa que los aspectos fuertemente coherentistas de su anterior formulación quedan incorporados en su pragmatismo actual.

Paso ahora a la tesis de la relatividad conceptual, con el fin de formular las tensiones que encuentro entre esta tesis de Putnam y su noción de verdad. Según este autor, la principal falla del realismo metafísico (o realismo externalista) es que no puede reconocer el fenómeno de la relatividad conceptual, y no lo puede reconocer "porque este fenómeno depende del hecho de que los mismos primitivos lógicos, $y$ en particular las nociones de objeto $y$ de existencia, tienen una multitud de usos diferentes, y no un 'significado' absoluto" (1987, p. 19). De aquí la famosa y controvertida afirmación de Putnam de que "los objetos no existen independientemente de los esquemas conceptuales". La idea del realista metafísico de que hay un uso de 'existir' que es inherente al mundo mismo, el cual nos permite plantear con sentido la pregunta: ¿qué objetos existen realmente?, es una mera ilusión.

Para el realista metafísico, ciertos enunciados se nos imponen porque "así es como son las cosas", su verdad se reconoce porque describen el mundo tal como es en sí mismo, independientemente de cualquier perspectiva. Y esto no sólo significa que algunos enunciados corresponden a la forma en que son las cosas, sino que además implica que la correspondencia entre lenguaje y mundo tiene que ser, ella misma, impuesta por el mundo. Pero éste es un supuesto incoherente, en opinión de Putnam, pues con base en la teoría de modelos sabemos que aun cuando determináramos los supuestos valores de verdad de nuestras oraciones, ello no determinaría una correspondencia única entre las palabras y los elementos del universo de discurso ( $f f r .1990$, p. 173). 
Ahora bien, el que los objetos no existan independientemente de los esquemas conceptuales implica que pueden existir teorías con ontologías incompatibles que sean ambas verdaderas. Según Putnam, su noción de verdad no nos obliga a tener que decidir entre ellas: "desde el punto de vista de la vida y de la práctica intelectual, una teoría que representa las interacciones físicas entre los cuerpos en términos de acción a distancia, y una teoría que representa la misma situación en términos de campos, pueden ser correctas las dos" (1990, p. 40). Esto simplemente quiere decir, en idea de Putnam, que diversas representaciones, diversos lenguajes, diversas teorías, son igualmente buenos en ciertos contextos. En esto consiste su pluralismo.

Pero entonces, ¿qué pasaría con una aseveración como "existen agentes causales que median la acción entre los cuerpos"? De acuerdo con el relativismo conceptual, si lo entiendo bien, la respuesta sería que esta afirmación es verdadera en una teoría y falsa en la otra, dado que estamos suponiendo que las dos teorías son "igualmente buenas". Esto es, se trataría de una aseveración que cambia su valor de verdad al pasar de una teoría a la otra, o dicho de otra manera, de una aseveración que tanto ella como su negación son ambas verdaderas. Si esto es así, ¿dónde queda la "idea clave" de la estabilidad o "convergencia" de la verdad? Recordemos que Putnam afirma que si tanto un enunciado como su negación pudieran justificarse, en las mejores condiciones que pudiéramos esperar, "no tendría ningún sentido pensar que el enunciado tiene un valor de verdad". ¿Qué sentido tendría entonces, en relación con aseveraciones como ésta, el viejo supuesto realista de que la verdad es una propiedad que "no se puede perder", supuesto que Putnam tanto se empeña en conservar y por el cual introduce la noción de idealización?

Me parece que la dependencia de nociones tan básicas como "objeto" y "existencia" respecto de las distintas versiones del mundo, o esquemas conceptuales, vuelve insostenible la idea de que la verdad es una propiedad estable de los enunciados. El realismo interno, con su tesis de la relatividad conceptual, parece no tener un lugar coherente para esta intuición del realismo metafísico.

Por otra parte, la teoría de la verdad como idealización descansa en el supuesto de que existe una noción de justificación que está por encima de los esquemas conceptuales. La frase "afirmar que un enunciado es verdadero es afirmar que podría ser justificado", apunta a un sentido de justificación que implica una noción muy fuerte de racionalidad. Con esto no quiero decir que Putnam quede comprometido con ciertos cánones o principios fijos, ahistóricos, que definan lo que es ser racional, cosa que rechaza tajantemente pues para él la racionalidad es algo que cambia y evoluciona con el tiempo. Sin embargo, el que las ideas de racionalidad y los modelos de justificación que de hecho utilizamos sufran cambios, y cambios profundos, no cancela para Putnam la necesidad de que haya un sentido de justificación que trascienda las distintas versiones del mundo. Esto se deja ver en un artículo donde Putnam discute las 
tesis de Kuhn, en el cual dice: "La idea de que hay una noción de justificación que es transcultural y, como dice Kuhn, no paradigmática - no simplemente una criatura de la epistemología local y de los estándares de la época-, es una idea correcta e importante" (1990, p. 125).

La importancia de esta idea se debe a que su negación implicaría, según Putnam, caer en la forma más común del relativismo que se autorrefuta. Pero scómo entender esa noción de justificación transcultural, y la noción de racionalidad que trae consigo? Me parece que Putnam da una pista cuando defiende la independencia de la justificación con respecto de la opinión mayoritaria, y afirma que la defensa de esta independencia no requiere de la postulación de cánones transhistóricos de justificación de las creencias, sino que lo único que se necesita es reconocer que la idea de que la justificación es independiente de la opinión de la mayoría "no es más que una propiedad del concepto mismo de justificación", es decir, "es una parte central de nuestra idea de justificación" (1990, p. 22).

El artículo sobre James, escrito con Ruth Anna Putnam, apoya la presunción de que Putnam daría un "argumento" similar para defender la necesidad de distinguir entre justificación y verdad (entre justificación "aquí y ahora" y justificación en condiciones ideales), y para explicar además el sentido de trascendencia cultural y temporal que encierra la noción de justificación ideal. Esto es, Putnam diría que la distinción entre justificación y verdad es deseable y necesaria porque constituye una parte central de nuestra idea misma de verdad, según la cual la verdad es algo más que la mera justificación de hecho; sin una noción semejante sería "vacío decir que lo que estamos tratando de hacer en la ciencia, o en cualquier otro lado, es encontrar la verdad" (1990, p. 222).

Esta línea de argumentación me plantea los siguientes problemas. En primer lugar, no me queda claro a quién se refiere el 'nosotros' implícito en frases como "es una parte central de nuestra idea de justificación", que usa Putnam con tanta frecuencia. Por un lado, la verdad como idealización parece remitir a un sujeto muy cercano al sujeto kantiano, es decir, cualquier "ser con una naturaleza racional y sensible". Pero, por otro lado, la diversidad de esquemas conceptuales desde los cuales se constituye el mundo, incluyendo a los sujetos mismos, parecería obligarnos a referirnos a los sujetos de cada esquema conceptual. De acuerdo con la primera opción, el 'nosotros' remitiría al sujeto de una racionalidad universal. Pero de acuerdo con la segunda, no queda claro cuál sería la extensión del 'nosotros', es decir, ¿dónde recortaría Putnam la comunidad que detenta las nociones de verdad, justificación y racionalidad, a las que él se refiere?

En segundo lugar, el argumento de que debemos postular la idea de justificación ideal porque es una parte constitutiva de nuestra noción de verdad, 
se aplica justamente con mucho mayor fuerza a la idea de correspondencia. Es cierto, como señala Putnam, que cuando se afirma que un enunciado es verdadero, se está queriendo decir algo que va más allá de la mera justificación de hecho. Pero ese algo más es precisamente que "así es como son las cosas", y no que "el enunciado sería justificable en condiciones ideales".

Luis Villoro ha destacado esta cuestión con mucha claridad: la pretensión de verdad no debe confundirse con la pretensión de justificación universal. La verdad no puede entenderse como un caso "ideal" de justificación objetiva. Una cosa es pretender que "si un enunciado es verdadero, el hecho enunciado es", y otra, muy distinta, pretender que "nadie puede acceder a razones que invaliden lo bien fundado de un enunciado" (Villoro, 1990, p. 83). Afirmar que un enunciado es verdadero es afirmar que "lo enunciado forma parte del mundo real, independientemente de que otros sujetos coincidan en enunciarlo" (ibid.). Para este autor, "la aceptabilidad racional en condiciones ideales puede ser un criterio estricto de objetividad, pero no corresponde a lo que entendemos por verdad" (ibid., p. 85). La noción de "verdad" está emparentada con la noción de "realidad", y "justificación" con "intersubjetividad", dice Villoro.

Me parece que el análisis que hace Villoro de las pretensiones de verdad bien podría, en principio, ser aceptado por un internalista, pues además de ser un análisis difícil de objetar, el aceptar que las pretensiones de verdad rebasan el terreno puramente epistémico —el ámbito de las razones-, y revelan un compromiso ontológico por parte de los sujetos, no nos obliga a aceptar la correspondencia metafísica, la correspondencia con un "mundo ya hecho", la cual es impuesta por ese mismo mundo. Esta aceptación, en cambio, sí nos permite darle un lugar a la idea de correspondencia, la cual está en la base de uno de los motores más efectivos del desarrollo científico: el ideal de alcanzar algo que está "ahí afuera" y conocerlo tal como es. De la misma manera que la idea de coherencia está en la base del ideal de sistematicidad completa entre nuestras creencias, o la idea de utilidad está en la base del ideal de predicción y control total de la naturaleza, la idea de correspondencia también ha cumplido una función heurística innegable en el desarrollo del conocimiento.

Ahora bien, Putnam con su idea de justificación ideal cree, en efecto, estar rescatando el núcleo de lo que entendemos por verdad. Incluso dice que su noción es una noción más "humilde" que la de correspondencia, y que es la noción que usamos constantemente tanto en la vida cotidiana como en la práctica científica (cfr. 1990, p. 40). Pero si Villoro tiene razón, como creo que la tiene, la noción de verdad de Putnam no se podría defender por esta vía. Y si, como sugerí, resultara que en efecto su noción de verdad entra en conflicto con su tesis de la relatividad conceptual, dicha noción tampoco podría preservar el supuesto de la verdad como una propiedad "que no se 
puede perder". En este caso, la postulación de la idea de justificación ideal se quedaría sin apoyo y sin función.

Me parece que la justificación ideal o idealizada más que recuperar "nuestras" intuiciones básicas sobre la verdad, recupera las fuertes intuiciones de Putnam sobre la justificación y la racionalidad; es un reflejo de su idea ('o su deseo?) de una racionalidad que atraviese las distintas versiones del mundo.

Ciertamente, un supuesto básico de su posición es que "la mente y el mundo hacen conjuntamente a la mente y al mundo" (1981, p. XI), pero al expulsar el "mundo" de la noción de verdad se deja todo el peso en la "mente". Putnam afirma, con toda razón, que "sin los valores cognitivos de coherencia, simplicidad y eficacia instrumental, no tenemos ni mundo ni hechos" (1990, p. 139), pero descuida filosóficamente el otro lado: sin mundo independiente no habría esquemas conceptuales, ni valores epistémicos, y mucho menos idea de verdad. El aceptar que no debemos cometer la "falacia de la división", y el reconocer que "la gloria de Kant [...] es decir que no es un desastre el mero hecho de que no podamos separar nuestra propia contribución conceptual de lo que está 'objetivamente ahî" (1987, p. 52), no implica negarle un lugar a lo que "está objetivamente ahî", al intentar dar cuenta de la verdad.

Lo que quiero decir, en pocas palabras, es que para dar cuenta filosóficamente de nuestras pretensiones de verdad se necesita destacar la intuición de que "la mente humana no ha creado las estrellas ni las montañas", pues de lo contrario difícilmente dichas pretensiones pasarían de ser meras inclinaciones u obsesiones. Por supuesto, como señala Putnam, esta "consideración elemental" de que la mente no ha creado el mundo, no es, ni de lejos, suficiente para resolver la cuestión filosófica del realismo versus antirrealismo (cfr. 1990, p. 30). Pero mi intención aquí no ha sido discutir el problema del "algo independiente", sino simplemente subrayar que la suposición de que éste existe es una parte central de lo que entendemos por verdad.

Desde luego, queda abierta la vía de cancelar el problema y decir que las pretensiones de verdad no constituyen un problema filosófico. Pero nada más lejos del pensamiento de un filósofo que pregunta: "icuál es el objeto de tener 'representaciones' [pictures], si no estamos interesados en ver qué tan bien reflejan lo que de hecho pensamos y hacemos?" (1990, p. 42). Es en este mismo espíritu que yo le preguntaría a Putnam si no sería más adecuado reformu- lar las pretensiones de verdad como pretensiones de correspondencia, y dejar la idea de "justificación en condiciones ideales" como expresión de una parte central de nuestra idea de racionalidad. Después de todo, cuando afirmamos que "hay mejores y peores justificaciones" lo que está inmediatamente en juego es nuestra idea de racionalidad, y no nuestra idea de verdad. Me pregunto si de esta manera no tendríamos un realismo con un rostro más humano. 
Bibliografía

Putnam, Hilary, 1981, Reason, Truth and History, Cambridge University Press.

—, 1987, The Many Faces of Realism, Open Court, La Salle (Ill.).

- 1990, Realism with a Human Face, Harvard University Press.

Villoro, Luis, 1990, “Sobre justificación y verdad: respuesta a León Olivé”, Crítica, no. 65 , México. 